\title{
PROKLA-Redaktion
}

\section{Editorial: Perspektiven der Gesellschaftskritik heute}

Das Verhältnis von Kapitalismus und Kritik ist ein politischer wie gesellschaftstheoretischer Dauerbrenner mit konjunkturellen Schwankungen, und es ist unbestreitbar, dass wir im Nachgang der Finanzmarktkrise im Herbst 2008 und der sich anschließenden Folgekrisen eine Kritik-Renaissance ungeahnten Ausmaßes und mit zum Teil wahrhaft erstaunlichen ProtagonistInnen erleben. Große Teile des eher kritikunverdächtigen Feuilletons verschrieben sich plötzlich (und mit kurzer Halbwertszeit) mit radikaler Rhetorik der Kritik des Kapitalismus: So war in der liberalen ZEIT (27.7.2009) zu lesen: „Der Kapitalismus ist genauso gescheitert wie der Sozialismus. Diese Krise ist das Symptom eines fundamentalen Wandels, es sind die Geburtswehen für eine neue Welt. So etwas hat in der Geschichte möglicherweise noch nie stattgefunden." Die Frankfurter Allgemeine Zeitung rief Kapitalismuskritik als neues heißes Thema aus und auch wenn die Titelüberschrift „We are all socialists now" des Magazins Newsweek (6.2.2009) ironisch grundiert ist, trifft sie doch den Geist insbesondere der ersten Monate nach der Insolvenz von Lehman Brothers. Systemkritik war plötzlich en vogue. Ginge es tatsächlich nur um eine feuilletonistische Kritikblase, wäre das zwar nicht uninteressant, aber doch nicht von nachhaltiger Bedeutung. Tatsächlich aber wurde das Jahr 2011 mit dem Arabischen Frühling, den heftigen, andauernden Protesten in
Griechenland, der spanischen Bewegung des 15. Mai, den Sozialprotesten in Israel (vgl. dazu PROKLA 166) sowie der Entstehung und Ausbreitung der OccupyBewegung zum Jahr der globalen Proteste. The protester avancierte gar zur vom Time Magazine gekürten Person des Jahres. Die mediale und politische Begeisterung für die Occupy-Bewegung reichte so weit in den Mainstream hinein, dass die Abwehr falscher Freunde schnell zur zentralen Übung der jungen Bewegung wurde.

Zwei Bücher, die unterschiedlicher nicht sein könnten, begleiteten die Proteste in den westlichen Metropolen und wurden in vielen europäischen Ländern zu unerwarteten Verkaufsschlagern: Zum einen Stephane Hessels Flugschrift „Empört euch“ - ein humanistisch grundierter, äußerst allgemein gehaltener Aufruf, sich einzumischen, ein Plädoyer gegen die Indifferenz der Massen, gegen soziale Ungleichheit, Finanzkapital und Rassismus. Obwohl der kurze Text analytisch nicht viel zu bieten hat, traf und trifft er ganz offenkundig einen Nerv der Zeit, wobei das Alter und die Biographie des 93-jährigen ehemaligen Résistance-Kämpfers, KZÜberlebenenden und Diplomaten für den Erfolg des Textes wohl entscheidend sind. Ganz anders das Buch „Der kommende Aufstand" des Unsichtbaren Komitees, das bereits seit 2007 in Frankreich und seit 2010 in deutscher Übersetzung große Aufmerksamkeit erlangte und dessen 
AutorInnen anonym - eben unsichtbar - bleiben. Getreu der Devise „Die Katastrophe ist nicht das, was kommt, sondern das, was da ist" werden mit anarchistischer Emphase Symptome des Zusammenbruchs der westlichen Demokratien zusammengetragen und in poetischer Sprache die Kraft gewaltsamer Aufstände beschrieben. ${ }^{1}$

Das in der Nachfrage nach so unterschiedlichen Statements seinen Ausdruck findende große Interesse an einer Kritik, die über das politische Alltagsgeschäft hinausweist, hat einen neuen Resonanzraum für theoretische Krisenanalysen und Gesellschaftsdiagnosen geschaffen. Seit Beginn der 2000er Jahre läßt sich eine beachtliche Zahl von Veröffentlichungen und AutorInnen feststellen, die der kritischen Gesellschaftstheorie, der politischen Theorie, der Kritik der politischen Ökonomie oder der feministischen und Queer-Theorie neue Impulse geben. Auch der Kritik-Begriff selbst ist mittlerweile zum Gegenstand einer Reihe von Veröffentlichungen geworden (vgl. Demirović 2008; Jaeggi/Wesche 2009; Forst u.a. 2009; Celikates 2009). ${ }^{2}$ Mit den Heften über „Marx und Foucault" (PROKLA 151, 2008), über „Sozialismus?“ (PROKLA 155, 2009), über „Marx!“ (PROKLA 159, 2010), über „Gesellschaftstheorie im An-

1 Stephane Hessel: Empört Euch, Berlin: Ullstein 2011; Unsichtbares Komitee: Der kommende Aufstand, Hamburg: Edition Nautilus 2010.

2 Alex Demirović (Hrsg.): Kritik und Materialität, Münster 2008; Robin Celikates: Kritik als soziale Praxis. Gesellschaftliche Selbstverständigung und kritische Theorie, Frankfurt/M. 2009; Rainer Forst u.a. (Hrsg.): Sozialphilosophie und Kritik, Frankfurt/M. 2009; Rahel Jaeggi, Tilo Wesche (Hrsg.): Was ist Kritik? Frankfurt/M. 2009. schluss an Marx" (PROKLA 165, 2011) hat auch die PROKLA zu dieser notwendigen Diskussion beigetragen. Dabei ging es der Redaktion darum, sich zwei komplementären Einseitigkeiten entgegenzustellen. Zum einen sollte das an Marx anschließende Projekt der Kritik der Gesellschaft nicht nur in diese Diskussion eingebracht werden, die erkennen lässt, dass sie diese Theorietradition weiterhin mit einem Tabu belegt, sondern es sollten auch seine Potenziale im Zusammenspiel mit neueren Ansätzen radikaler Gesellschaftskritik reflektiert werden. Zum anderen sollte deutlich gemacht werden, dass die Kritik der politischen Ökonomie und die daran anschließende Tradition der Gesellschaftstheorie - gerade in Zeiten brachialer (nicht nur) feuilletonistischer Verkürzungen Marx'schen Denkens - nicht auf eine bloße Kritik des Wirtschaftssystems und der entfremdenden Macht des Markt reduziert werden darf.

Mit dem vorliegenden Heft 167 weiten wir den theoretischen Blick und wenden uns gesellschaftskritischen Perspektiven nach dem cultural und discursive turn zu: Im Spannungsfeld von Postmarxismus und Poststrukturalismus, von Cultural Studies und Kritischer Theorie, von feministischer Kritik und theoretischen Grenzgängern wie Cornelius Castoriadis fragen die AutorInnen dieses Heftes, ob und inwiefern wir mit, gegen und über diese Perspektiven hinaus das Projekt der Gesellschaftskritik als kritische Praxis in den aktuellen Konstellationen denken können. Wie verhalten sich Theorie, Kritik und Praxis zueinander? Wo finden aktuelle gesellschaftskritische Ansätze ihre theoretischen wie praktischen Ansatz- und Ankerpunkte? Wie können im Lichte der seit drei Jahrzehnten aus unterschiedlichen Perspektiven entwickelten Wahrheits-, Subjekt- und Universalitätskritik(en) 
kritische Standpunkte eingenommen und ausgewiesen werden? Welche Anschlüsse an materialistische Kritiken bieten sich an? Was bedeutet Gesellschaftskritik für theoretische Ansätze, die „Gesellschaft“ als Bezugsgröße problematisieren und an die Stelle der "großen Ordnung“ und Teleologie ein dezentrisches und anarchisches Weltbild setzen? Und wie sind die aktuellen Entwicklungen in den Bewegungszonen zwischen Akademie und Politik zu bewerten, die - in der gegenwärtigen „Krise des Kapitalismus“ sicher nicht zufällig - auf eine Suche nach Denkangeboten hinauslaufen, die über Wahrheits-, Universalismus- und Subjektkritik hinausweisen?

Immer wieder wird der Vorwurf vorgebracht, dass die Gesellschaftsanalysen und Kritiken, die aus den Protestbewegungen der 1960er und 1970er Jahre hervorgegangen sind, bei all ihrer Radikalität von der neoliberalen Strategie vereinnahmt und genutzt worden seien, um mit ihnen den Kapitalismus zu revolutionieren. In der Folge sei von den emanzipatorischen Gehalten dieser Theorien wenig oder gar nichts übrig geblieben - wenn sie jemals welche hatten. Tatsächlich wird heute auch von denen, die sich dem Poststrukturalismus oder Postmarxismus zurechnen, darauf hingewiesen, dass die theoretische Emphase für das Fluide und Nicht-Fixierbare ebenso wie der antiinstitutionelle Gestus und der Fokus auf Prozesse der Subjektivierung Anschlüsse an Flexibilisierung, De-Regulierung und Entstaatlichung im Neoliberalismus bieten können. Wir verstehen die PROKLAHefte demgegenüber als Anstoß zu einer „rettenden Kritik“, sofern diese überhaupt notwendig ist. Denn wir vermuten, dass die Theoriebildung der späten 1960er Jahre und die davon ausgehenden Impulse ein Kritikprogramm hervorgebracht hat, das immer noch unabgegolten ist. Entspre- chend fragen verschiedene AutorInnen dieses Heftes danach, ob und inwiefern eine solche Entwicklung durch die angeführten Theorien sowie die an sie anschließenden Bewegungen ermöglicht wurde - und wie der Gefahr der ungewollten Vereinnahmung zu entgehen ist. Konsequenterweise steht damit die - unterschiedlich gestellte und beantwortete - Frage im Zentrum des Heftes, wie im Sinne einer radikalen Gesellschaftskritik die Problematisierung von Subjekt(ivierung), gesellschaftlicher Totalität und Wahrheit mit einer kapitalismuskritischen Thematisierung sozialer und ökonomischer Fragen zusammenzuführen ist.

Silke van Dyk greift in ihrem Beitrag das vielfach artikulierte Unbehagen ob der Analysekraft und des Kritikpotenzials von Poststrukturalismus und Cultural Studies auf, und konfrontiert das theoretische Paradigma mit Fragen nach Kritik und Wahrheit, nach Ungleichheit und Herrschaft sowie nach der Analyse von Gesellschaft als „großem Ganzen“. Das gegenwärtige gesellschaftskritische Defizit des Poststrukturalismus kann, so ihre These, durch Denkfiguren des Paradigmas selbst eingeholt werden. Dafür sei es jedoch notwendig die partielle „Selbstdestruktion " und Entpolitisierung poststrukturalistischen Denkens zu überwinden, die im Zuge seiner akademischen Popularisierung zu beobachten sei.

Hanno Pabl sammelt in seinem Beitrag Indizien dafür, dass es für eine avancierte Gegenwartsanalyse kapitalistischer Vergesellschaftung ergiebig ist, bestehende Spannungen zwischen Poststrukturalismus und Ökonomiekritik als Forschungsheuristik fruchtbar zu machen. Konkret zeigt Pahl auf, dass und wie eine genealogisch-poststrukturalistische Ökonomiekritik als Korrektiv modernisierungstheoretischer bzw. subsumtionslogisch verfahrender 
Lesarten Marxscher Ökonomietheorie eingesetzt werden und dazu beitragen kann, die historische Trennschärfe sowie das Kontingenzbewusstsein kritischer Kapitalismustheorien zu vergrößern.

Barbara Umrath fragt danach, ob die seit geraumer Zeit zu beobachtende Integration feministischer Forderungen in den politischen Mainstream als Erfolg der Kritik oder eher als eine Art „feindliche Übernahme" zu verstehen ist. In kritischer Auseinandersetzung mit Analysen zur Vereinnahmung feministischer Kritik diskutiert die Autorin, wie es zur Umdeutung feministischer Positionen kommen konnte und wie diese in Zukunft zu verhindern sind. Zentrale Annahme ist, dass der Feminismus nur dann seinen gesellschaftskritischen Impetus zurückgewinnen kann, wenn er sich wieder stärker politökonomischen Entwicklungen zuwendet. Theoretisch empfiehlt die Autorin einen Anschluss feministischer Kritik an die frühe Kritische Theorie.

Robin Mohan und Daniel Keil entwickeln eine materialistische Kritik der Anerkennungstheorie Axel Honneths. Mit der engen Anbindung der Anerkennungstheorie an das Institut für Sozialforschung werde - so die Autoren - suggeriert, dass ein kategorialer Rahmen entwickelt worden sei, der es ermögliche, die auch heute aufrecht zu erhaltenden Ansprüche der Kritischen Theorie besser zu verwirklichen. Mohan und Keil argumentieren stattdessen, dass die kategorialen Umstellungen Honneths der zentralen Aufgabe kritischer Gesellschaftstheorie - die kapitalismusspezifische Form von Herrschaft und die Verhinderung von Emanzipation zu begreifen - nicht gewachsen sei. Damit, so die These, wird Honneth seinem Anspruch nicht gerecht, den Zusammenhang von kritischer Theoriebildung und verändernder Praxis zu restituieren.
Harald Wolf wiederum zeigt auf, dass das Werk des theoretischen Grenzgängers Cornelius Castoriadis einen wertvollen Beitrag zur Neuorientierung kritischen Denkens und emanzipatorischen Handelns leisten kann. Castoriadis eröffne einen neuen Blick auf die Bedingungen und Möglichkeiten von Gesellschaftskritik und setze in der Kapitalismusanalyse und -kritik andere Akzente als gegenwärtig dominierende Spielarten der Analyse und Kritik: Während diese die Kapitalismuskritik primär als Marktkritik konzipierten, begreife Castoriadis den Kapitalismus als Organisations-, Rationalisierungs- und Kontrollprojekt. Daraus folge schließlich sein ausgeprägtes Interesse an Widerstand und praktischer Kritik im Alltag, die als Keime autonomer Vergesellschaftung gedeutet werden.

In den Debatten um Perspektiven radikaler Gesellschaftskritik spielt die im weiteren Sinne postmarxistische politische Philosophie derzeit eine herausragende Rolle, weshalb zentrale Referenzautoren dieses Feldes - so Jacques Rancière, Antiono Negri, Alain Badiou oder Slavoj Žižek - in diesem Heft nicht fehlen sollen. Daniel Loick beschäftigt sich mit Jacques Rancières Beitrag zur Frage der intellektuellen Emanzipation. Rancière hat in seinen frühen Arbeiten eine radikale Kritik an den disziplinierenden Effekten staatlicher Bildung vorgelegt, die provokanterweise auch eine anarchistische Zurückweisung fortschrittlicher Reformprojekte beinhaltet. In kritischer Reflexion von Rancières Prinzip der „axiomatischen Gleichheit“ kommt Loick zu dem Schluss, dass Rancières anti-institutionelle Kritik am Bildungssystem zwar zu spontaneistisch und voluntaristisch ist und dass sie Gefahr läuft Prozessen neoliberaler De-Regulierung und Entstaatlichung in die Hände zu spielen, dass sie aber dennoch instruktiv für 
gesellschaftskritische Reflexionen gängiger Bildungspraktiken ist.

Thomas Seibert vermisst in seinem Beitrag das Spannungsfeld von poststrukturalistischen und postmarxistischen Positionen und geht der Frage nach, wie ein Subjekt der Kritik zu restituieren ist. Während poststrukturalistische Theoretiker wie Deleuze/Guattari und Foucault den Tod des Menschen verkündeten und den teleologischen Geschichtsverlauf des traditionellen Marxismus in einer pluralen Geschichte der Kontingenzen aufösten, versuchen Postmarxisten wie Hardt/Negri, Badiou und Žižek zu einem historischen Subjekt und einer materialistischen Teleologie zurückzukehren. Seibert erörtert, inwiefern diese Kritikbewegungen keinen „Rückfall“ hinter poststrukturalistische Problematisierungen darstellen, eröffne sich mit der Perspektive des „Posthumanismus" doch ein dritter Weg jenseits einer fruchtlosen Entgegensetzung von Poststrukturalismus und Postmarxismus.

Außerhalb des Schwerpunkts untersucht Anne Steckner die islamische AKP, die seit 2002 in der Türkei mehrere Wahlerfolge feiern konnte und inzwischen eine hegemoniale Stellung erreicht hat. Indem die Autorin die Politik der AKP in mehreren Feldern untersucht, macht sie deutlich, dass es sich hier um eine eigentümliche neoliberal-islamische Synthese handelt, die sich mit einer Mischung aus Konsens und Zwang eine unerwartet stabile Herrschaftsposition verschafft hat.

Dieses Heft wurde mit viel Engagement von Silke van Dyk als Gastredakteurin konzipiert, organisiert und inhaltlich gestaltet. Dafür danken wir ihr sehr herzlich.

Am 28. April fand in Berlin die diesjährige Mitgliederversammlung der Vereinigung zur Kritik der politischen Ökonomie statt. Die Vereinigung gibt die PROKLA heraus und wählt Redaktion und Redaktionsbeirat. Unser Redaktionsmitglied Martin Kronauer stand aufgrund beruflicher Belastungen für eine erneute Wahl leider nicht mehr zu Verfügung. Allerdings bleibt er der PROKLA weiterhin eng verbunden, er wurde er in den Redaktionsbeirat gewählt. Ebenfalls aus Zeitgründen kandidierte Klaus Müller nicht mehr für den Beirat. Beiden sei an dieser Stelle für ihre bisherige Arbeit ganz herzlich gedankt. 